\title{
Comparing the settlement about prediction of the soft soil foundations
}

\author{
Cui Zhiyong, ${ }^{1, \mathrm{a}}$, Qing $\mathrm{Wei}^{2, \mathrm{~b}}$, Sun Shiwei ${ }^{3}$, Li Haibin ${ }^{4}$ \\ ${ }^{1}$ The preparatory office of upgrading and expansion about Shi'an \\ Expressway, China \\ ${ }^{2}$ School of Materials Science and Engineering, Chang'an University, \\ Xi'an 710064, China; \\ ${ }^{3}$ School of Materials Science and Engineering, Chang'an University, \\ Xi'an 710064, China; \\ ${ }^{4}$ Department of Architecture and Civil Engineering ,Xi'an University of \\ Science and Technology, \\ Xi'an, 710054, China; \\ a782532634@qq.com, ${ }^{b}$ lihaibin1212@126.com
}

\begin{abstract}
.
In this paper, the index curve model and hyperbolic model are analyzed. And the settlement value of a real project is forecasted based on these models. The research show that the index curve prediction results relatively conservative. And the cross section of subsidence prediction shows that there is a large difference between these two kinds of model. But the stability of predicting is nearly the same.

Keywords: Settlement, index curve forecasting model, hyperbolic forecasting model.

\section{Introduction}

In the upper loads, Foundation settlement is the deformation due to the compression function .In the process of the compression, foundation settlement contains some processes that the tiny water discharged ,the void fraction reduced and the soil particles adjust and rearranged. Therefore, the cause of deformation and subsidence and the soil properties is the main factors influencing the foundation settlement. As the classical method of settlement calculation, the
\end{abstract}


above two factors make the following processing:(1) based on the theory of semi-infinite space have identity each other ,we can calculate additional stress field of the foundation;(2) Based on one dimensional compression test, we can measure the compressibility of soil, and use the hierarchical method to calculate the settlement.

However, with the gradually increase of people's understanding of foundation settlement, the control of the settlement constantly change from after testing to before work. On the basis of settlement mechanism and the practical engineering, there are two types of the prediction model about foundation settlement. These are mathematical prediction method based on mathematical and mechanical model, and the prediction method is based on settlement observation. Because the mathematical prediction has some advantages of simple, intuitive few parameters and easy to obtain data, some researchers all have recognized it .Mathematical prediction have the larger subjectivity ,it have a bigger error at real engineering and can not meet the demand of engineering .Prediction based on observation data is a traditional settlement prediction. According to the soil consolidation and constitutive relation, we can establish a model. Using the settlement data to calculate the parameters of foundation soil and have a settlement prediction. Although this method is relatively complicated, the result is relatively accurate and have a widely engineering application .For a highway that is soft soil and have a bigger silt content, this paper have a analyzed with the index curve and hyperbola method .

\section{Model of Prediction}

\section{(1)Model of exponential curve prediction}

Exponential curve assume that the average speed of settlement reduce as the form of exponential curve decreases and the exponential curve $\lg (\Delta S / \Delta t)$-t is three line with bent in Fig. 1.According to the experience, the public expressed as follows: 


$$
S_{t}-S_{0}=\left(S_{\infty}-S_{0}\right)^{e^{(t-t) / \eta}}
$$

Choosing (t1, S1) and $(\mathrm{t} 2, \mathrm{~S} 2)$ calculate the $\eta$ in the straight line of settlement .So we can calculated the final settlement :

$$
S_{\infty}=S_{0}+\eta(\Delta S / \Delta t)_{0}
$$

$S_{0}$ is flexible point of settlement curve , $S_{\infty}$ is final value of settlement and $(\Delta S / \Delta t)_{0}$ is sedimentation rate in the flexible points.

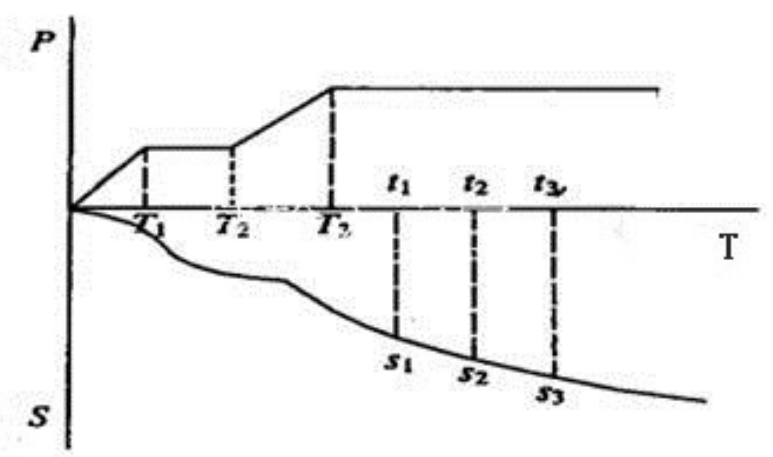

Fig. 1. line of bent

\section{(2) The model of hyperbolic}

If use the hyperbolic in the flexible point ,we can have a final prediction of settlement value.

$$
S=S_{0}+\frac{t}{A+B \times t}
$$


$S$ is the final settlement value, $S_{0}$ is the settlement value in the flexible point and $t$ is the interval of time, A and B are fitting coefficient of curve .The formula can be expressed as follows. And the method to calculate A and B are showed in Fig. 2.

$$
\frac{t}{S_{t}-S_{0}}=A+B t
$$

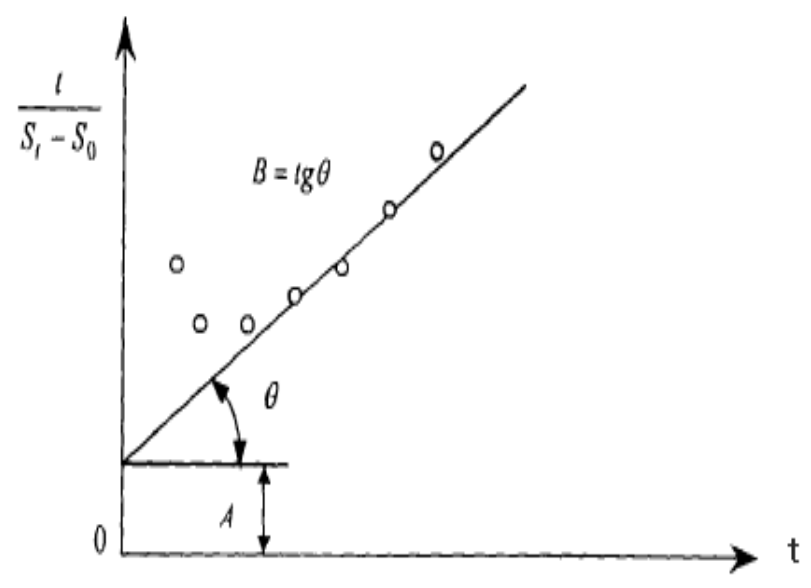

Fig. 2. The calculation of hyperbolic type A and B

\section{An Engineering Example}

The length of a highway is $45 \mathrm{~km}$, its terrain is spacious. There are many ponds, and the soft soil foundation account for about $92 \%$ of the total length of sub grade. The height of soft soil foundation is more than $20 \mathrm{~m}$ and the thickest place is $33 \mathrm{~m}$. It is silt which the characteristic is low intensity, high compressibility, and slow consolidation and so on. After paving and tracking observation about 3 years [9], testing 12 sedimentation points and fitting the sedimentation is more than $500 \mathrm{~mm}$ and the dot pitch is more than $200 \mathrm{~m}$. 


\section{The Prediction Results and Analysis}

\section{The Final Sedimentation}

The final sedimentation is through the exponential curve fitting and hyperbolic fitting. Obviously, compared two kinds of curve fitting, we can get the results that have large differences. Comparing with the observed values, the final prediction results can increase. Because the observed values are short-term while the prediction results are long-term, even the whole lifecycle of the situation of sub grade sedimentation also increases. Comparing the prediction results of the exponential curve and hyperbolic, the prediction results of the exponential curve are conservative. But the accuracy of the results of two kinds prediction results and the observed values need long time to prove.

\section{Error comparison}

This paper uses the average forecast deviation to analyze the stability of the prediction results of the two types of models. The result shows in Fig. 4. We can find:

(1) The average deviation has large difference in the place of different observation. For example, the forecast deviation at point 4 is 8 times than point 8 , and point 8 is more stable.

(2) Comparing the same observation point, we can find that the results are similar. 


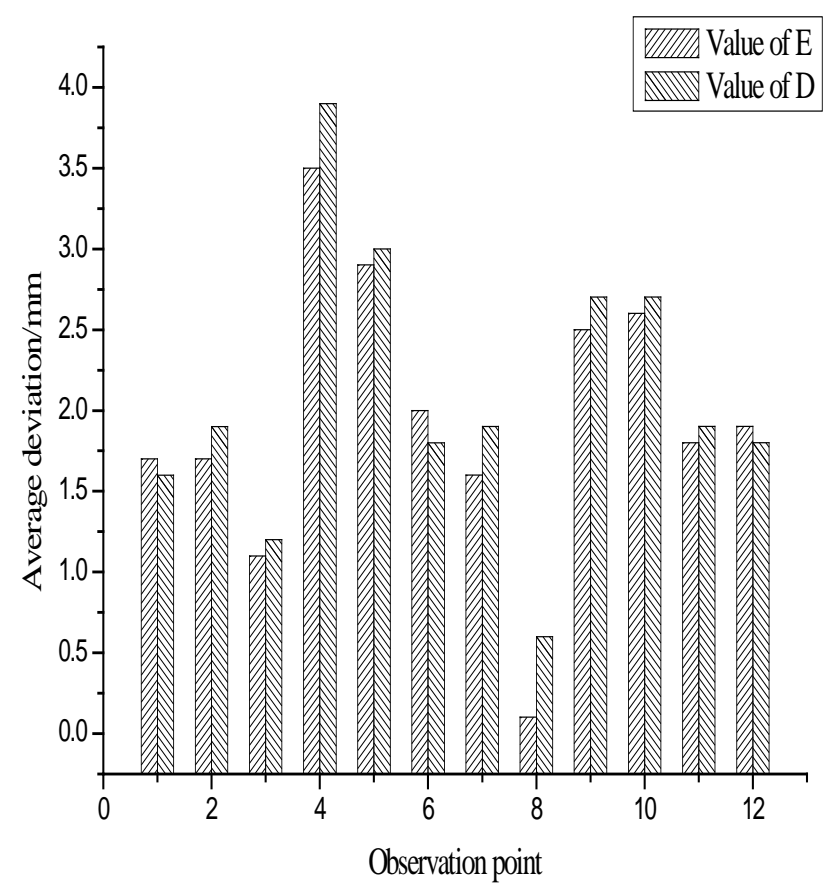

Fig. 3. Analyzing the average forecast deviation

( $\mathrm{E}$ is the prediction exponential curve, $\mathrm{D}$ is the prediction hyperbolic)

\section{A Typical Cross Section}

Fig. 5 shows the prediction curve of the cross section sedimentation at point 6 :

(1) The result of sedimentation in 200d and the results of observed values are extremely consistent. Because the prediction model is based on observations, obviously it is scientific.

(2) After 100d, the prediction of index model and prediction of hyperbolic model has many differences. Two kinds of prediction methods have different applicability in different working condition. But the specific situations need more researches. 


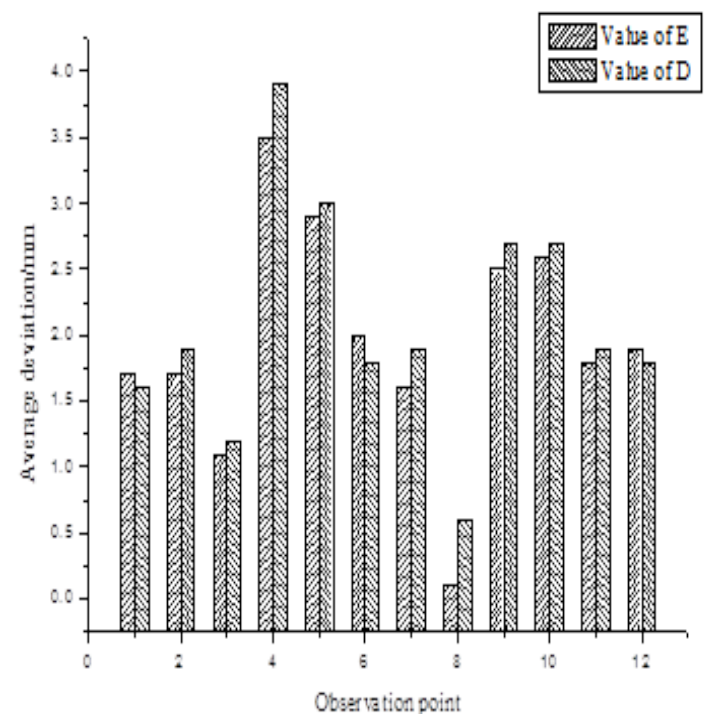

Fig. 4. Analysis of the forecast of the average deviation ( $\mathrm{E}$ is the index curve prediction, $\mathrm{D}$ is the hyperbolic prediction)

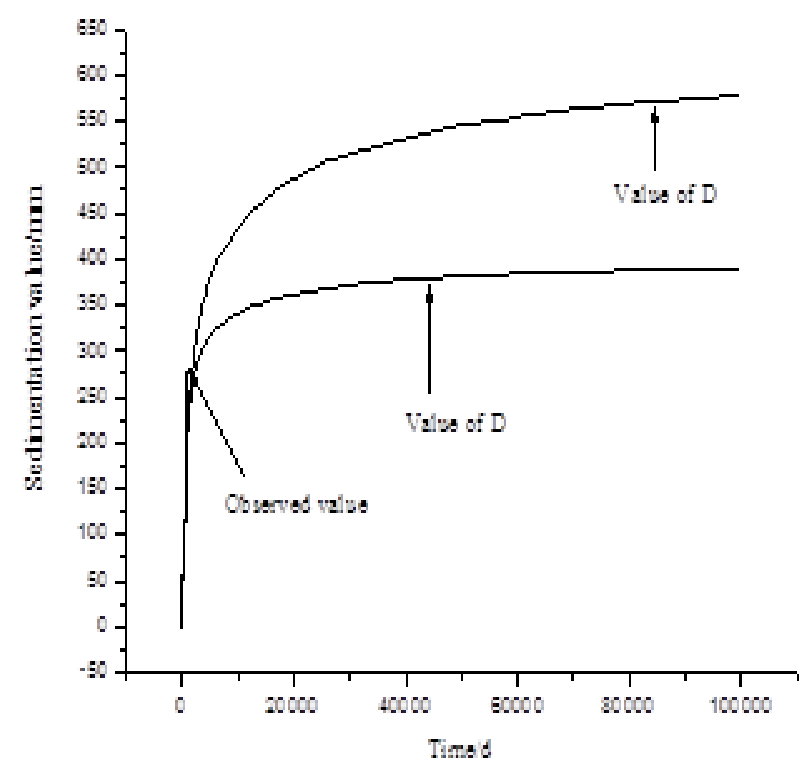

Fig. 5 Analysis of typical cross section

( $\mathrm{E}$ is the index curve prediction, $\mathrm{D}$ is the hyperbolic prediction) 


\section{Conclusions}

(1) Comparing the prediction results of the exponential curve and hyperbolic, the prediction results of the exponential model are conservative;

(2) The late results of the exponential model and hyperbolic model have large differences;

(3) The adaptation of two kinds of prediction model has a further observation.

\section{References}

[1] Xu Hua, Application of BP neural network in prediction of soft soil foundation

settlement in highway [J].Science technology and Engineering,2006,6(16):2552-2555.

[2] Song Xuguo, Liu Yuanfeng, Yang Mingyu, Using the observed data of settlement calculation of subsidence in future applications in Qinhuangdao Shenyang Dedicated Passenger Line[J].Subgrade Construction,2002,(5):37-40.

[3] Cao Guoqiang, Zhang Yiping, Zhang Tuqiao, The relationship between the rate and the settlement of the foundation settlement and its application[J].Rock-Soil Mechanics, 2003,24(3): 467-470.

[4] Gan Youwen, Wang Zhiliang, Zheng Hua, Modified hyperbolic model in prediction of foundation settlement[J]. Hydrogeology and Engineering Geology, 2004,(1):98 -100.

[5] Lv Xiujie, Study on prediction model of settlement of Soft Subgrade after[J]. Rock-Soil Mechanics, 2009, 30(7):2091-2096. 